\title{
Poly(ADP-ribose) polymerase-1 activity modulates mitochondrial function following UVB irradiation
}

Csaba Hegedüs ${ }^{1}$, Gábor Boros ${ }^{3}$, Eszter Anna Janka ${ }^{1}$, Marianna Lovászi ${ }^{1}$, Katalin Karikó ${ }^{3}$,

Tamás Juhász ${ }^{2}$, Gréta Kis Nikoletta ${ }^{2}$, Gabriella Emri ${ }^{1}$, Péter Bai ${ }^{4}$, Éva Remenyik

1. Department of Dermatology, University of Debrecen, Debrecen, Hungary

2. Department of Anatomy, Histology and Embryology, University of Debrecen, Debrecen, Hungary 3. RNA pharmaceuticals, BioNTech AG, Mainz, Germany

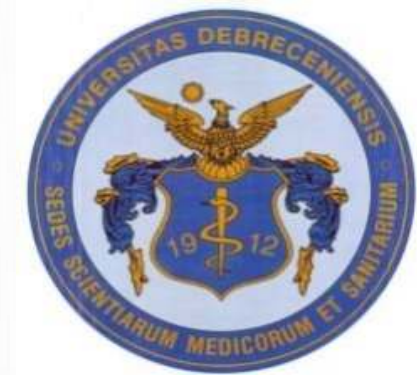

4. Department of Medical Chemistry, University of Debrecen, Debrecen, Hungary

5. Momentum program of the Hungarian Academy of Sciences

\section{Introduction}

Exposure of skin to ultraviolet radiation is the main cause of skin cancer development. Among UVR, UVB induces diverse cellular function and promotes the formation of DNA helix distorting photolesions such as (6-4) photoproducts and cyclobutane pyrimidine dimers (CPD). Effective repair of such lesions by the nucleotide excision repair pathway is required to prevent DNA damage and mutations. Poly(ADP-ribose) polymerase-1 (PARP-1) is a zinc finger protein and a key mediator in several cellular processes. It rapidly consumes cellular NAD and by that way supresses the activity of mitochondrial regulators ultimately leading to impaired mitochondrial function.

To investigate the impact of PARP inhibition on different mitochondrial parameters, human immortalized keratinocytes (HaCaT) were treated with $25 \mu \mathrm{M} \mathrm{ABT}-888$ and with a single dose of 20 and $40 \mathrm{~mJ} / \mathrm{cm}^{2}$ of UVB.

\section{Results}

PARP-1 inhibition reduced cell viability coupled with inefficient removal of CPDs and DNA strand breaks $\left(20 \mathrm{~mJ} / \mathrm{cm}^{2}\right)$
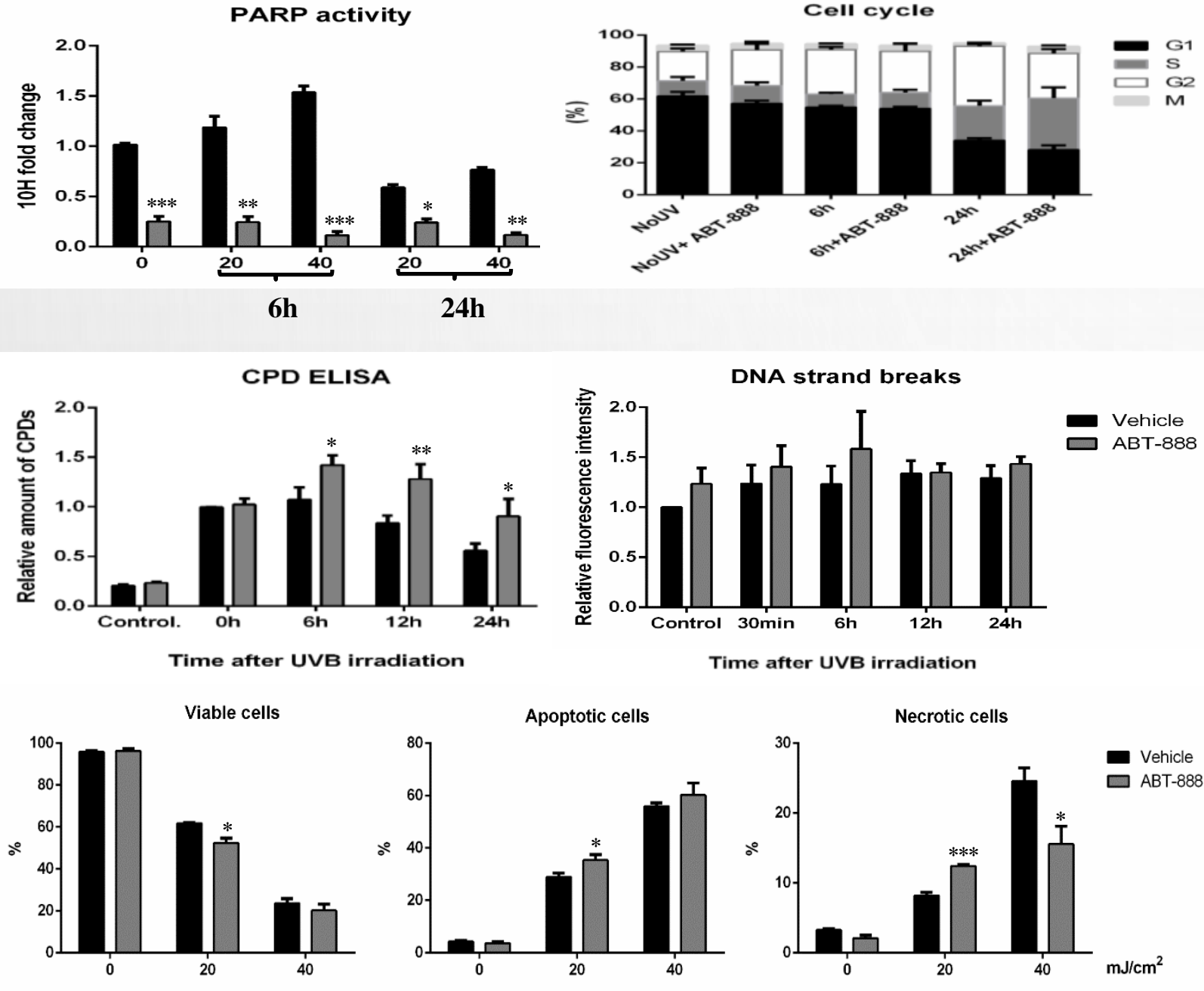

PARP inhibition and UVB induces mitochondrial morphological changes $(24 \mathrm{~h})$
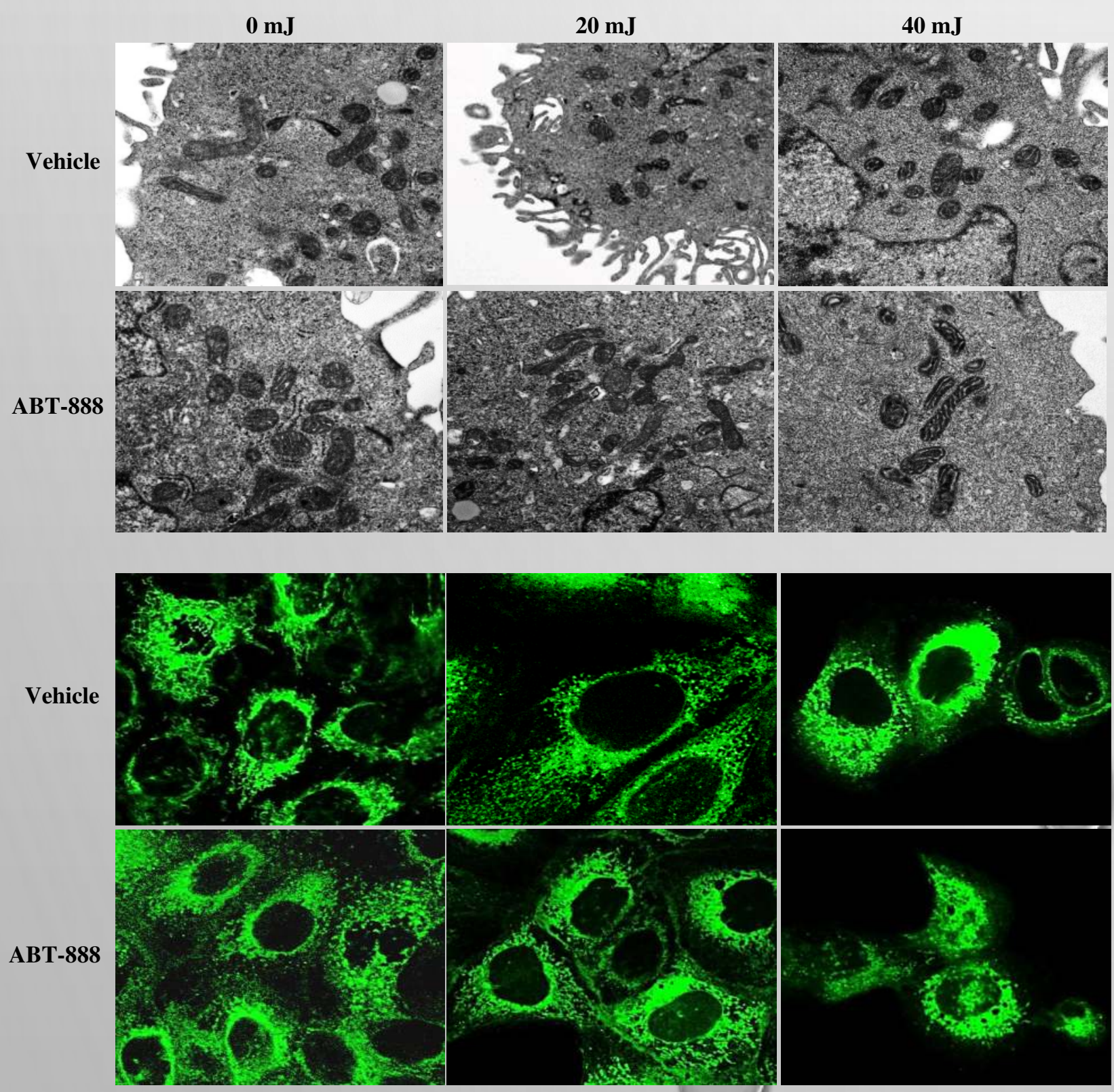

OIKA
PARP-1 regulates mitochondrial number, area, mass and mtDNA content (24h)
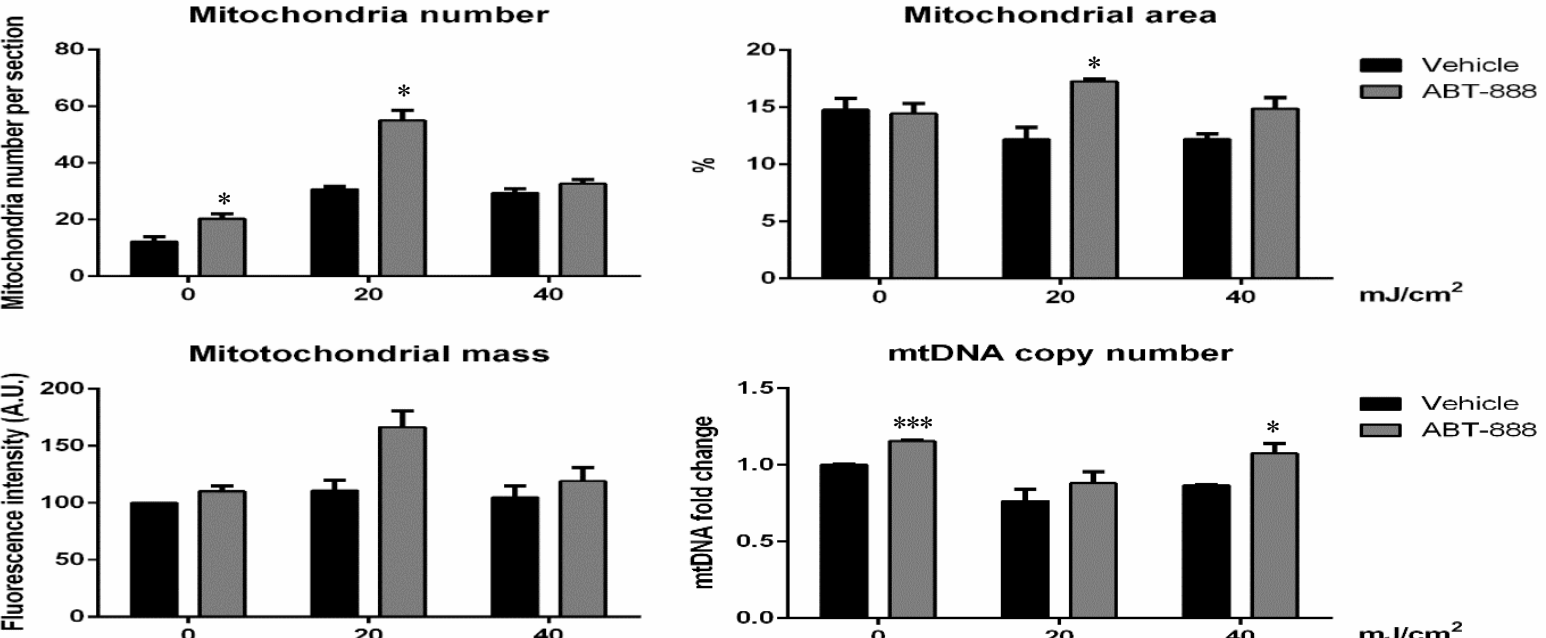

PARP inhibition induces mitochondrial depolarization and late increase in ROS production (24h)

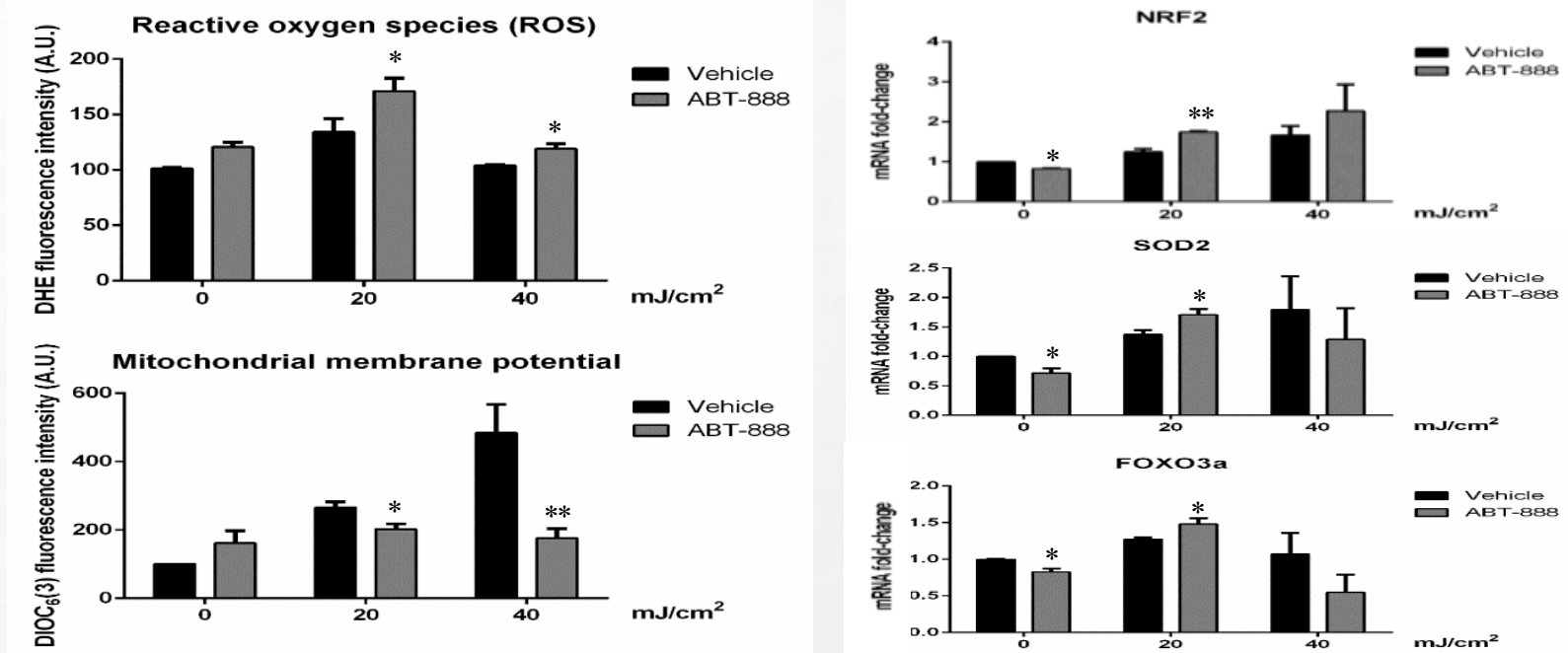

Elevated ATP level is dependent on oxidative phosphorylation (24h)

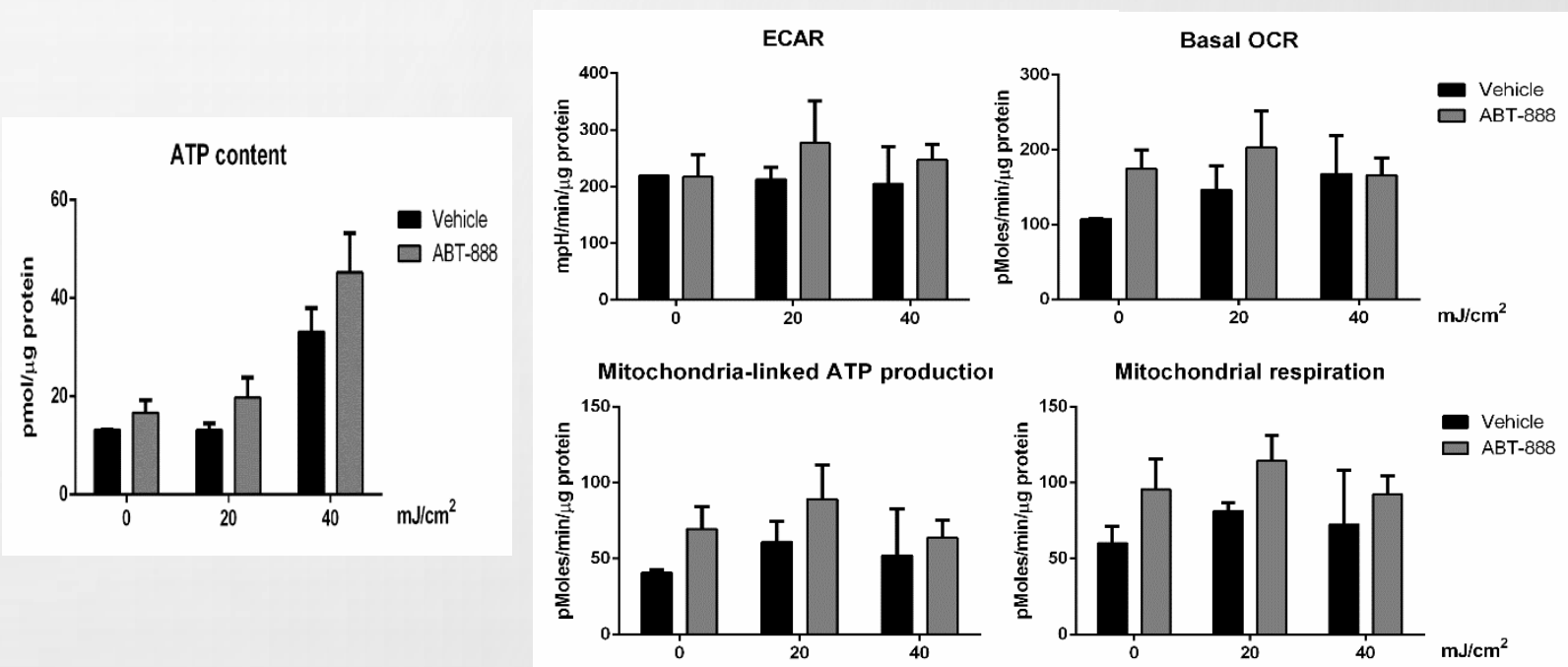

Cellular NAD ${ }^{+}$contributes to gene expression changes of mitochondrial regulator (24h)
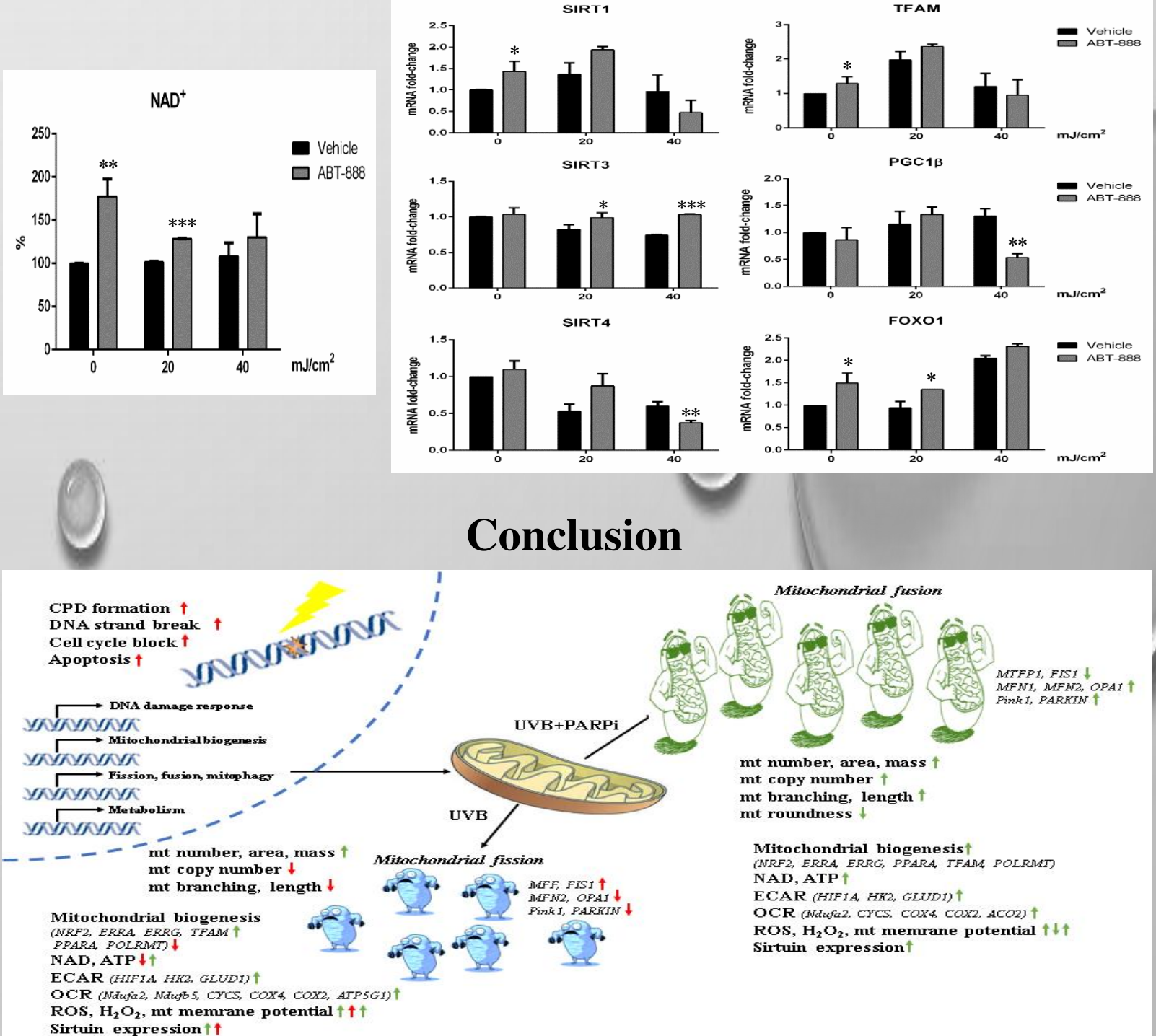

Our work was supported by: NKFIH K108308, C129074, OTKA 